\title{
Innovative and Timely Approaches to Suicide Prevention in Medical Education
}

\author{
Christine Yu Moutier ${ }^{1}$ (D)
}

Received: 25 March 2021 / Accepted: 8 April 2021/Published online: 5 May 2021

(C) Academic Psychiatry 2021

There has never been a timelier moment to focus on suicide prevention. Science is providing new answers about risk and prevention, the public is more interested than ever in mental health and suicide prevention, and the COVID-19 pandemic has only furthered the need to protect the resilience of patients and health professionals. The June 2021 issue of Academic Psychiatry features innovative approaches to training related to suicide prevention and support for trainees.

Public interest in suicide prevention has been growing exponentially. Attitudes toward mental health and suicide were already evolving in positive ways pre-pandemic: more than $90 \%$ of US adults viewed suicide as generally preventable and mental health as or more important than physical health [1]. During the pandemic, attitudes shifted toward an even greater sense of urgency and concern about suicide. Twenty-five percent of Americans reported accessing mental health care in some way during the pandemic, including through technological and virtual means [2]. An unprecedented number of legislative acts related to mental health and/or suicide prevention have been passed into law in 2020: 5 federal and 27 state laws [3], which speak to advances in the science and many people speaking out, elevating the experiences of suicide loss and lived experience, culminating in tremendous political will and culture change. In short, especially now during the COVID-19 pandemic, the USA has great societal resolve to address this leading cause of death [4].

But even while US culture has been opening up to the critical importance mental health plays in life, the national suicide rate has been increasing, by 35\% from 1999 to 2018 [5]. On December 22, 2020, the US Centers for Disease Control and Prevention released 2019 data showing the first year-over-year decrease in 20 years [4]. Thus, during the period when attitudes were progressing and stigma diminishing,

Christine Yu Moutier cmoutier@afsp.org

1 American Foundation for Suicide Prevention, New York, NY, USA suicide rates were rising; attitudinal shifts are important, even necessary, but clearly not sufficient to prevent suicide. Public interest must progress to action - in the form of implementation of interventions that have been demonstrated to save lives. These interventions, developed for community and clinical settings, need to be scaled widely enough to reach those whose risk may be escalating, an ever-changing subgroup within the larger population.

Interventions can also be developed for various points along the continuum of suicide risk, from universal primary prevention efforts to more targeted approaches even during the moment of acute crisis. Upstream interventions in suicide prevention refer to universal approaches that, like the Good Behavior Game, a classroom management system for early elementary school, can increase protective factors like coping and self-efficacy and reduce risk factors that later could increase suicide risk [6]. Targeted interventions provide opportunities for risk reduction by identifying individuals with higher suicide risk and providing them with specific interventions. Only in recent years has the field of suicide science developed and evaluated such approaches, producing a body of new tools and evidence, growing each year.

A nationwide initiative called Project 2025 [7] focuses on implementation at a broad scale, with the ultimate goal of decreasing the US suicide rate by $20 \%$ by 2025 . The American Foundation for Suicide Prevention conducted a modeling exercise based on the best suicide preventive science, which pointed to three settings where missed opportunities could be replaced with evidence-based risk-reducing interventions to save the most lives: health systems (e.g., primary care, emergency departments, and behavioral health), correctional settings, and firearm-owning communities. Health care settings loom large in this data-driven suicide prevention initiative, as the $20 \%$ by 2025 goal can only be reached through health system change and by training health care professionals in the best ways to detect and reduce suicide risk. 


\section{Training for Suicide Preventive Patient Care}

Those who are privileged with the role of educating physicians and other clinicians have a special responsibility to advance suicide prevention in training. Every suicide prevention training for teachers, first responders, and family members ("gatekeeper trainings") teaches people to bring an individual in crisis to a health care provider for evaluation and treatment. And yet, what do many people encounter when they present in crisis to an emergency department, primary care office, or even a mental health setting? It is often not what health care providers would want for their own family members: fractured health systems, problems accessing care, overreliance on hospitalization due to a lack of alternate resources, demeaning or culturally inappropriate experiences in health settings - the very place where healing should be a priority. Health professionals and leaders must do their part to equip the health care workforce (and build health systems) that are conversant and compassionate in suicide prevention, able to detect suicide risk (even without the overt presentation of suicidal thoughts), and knowledgeable about evidence-based brief interventions and treatment approaches.

The time is right because the evidence for what constitutes effective suicide preventive patient care is mounting [8]. The field of suicide science is burgeoning with new discoveries in areas such as genetics, neurobiology, clinical interventions, lethal means reduction, policy, and programs based in community, family, and school settings. Additionally, it was only recently in 2018 that the field of suicide prevention reached consensus on the minimum standards of care related to screening, risk assessment, and brief interventions to reduce suicide risk in primary care, emergency departments, and mental health care settings [9]. Although it is notable and regrettable that so few medical schools and residency curricula have included formal education on suicide [10], it is encouraging that enough evidence is now available to implement training with greater confidence.

In the June 2021 issue of Academic Psychiatry, several articles highlight ways to integrate suicide prevention into medical education [10-14]. Some of these articles detail novel curricula and their outcomes, which could be replicated as authors have offered to provide the teaching materials. Other articles focus on postvention following patient suicide [15-18], a critical part of suicide prevention education and a way to support and protect trainees after future loss, and have a critically important focus featuring suicide prevention initiatives for trainees and faculty.

\section{Preventing Suicide Among Health Professionals}

We can no longer accept as status quo that suicide rates are higher among health professions compared with many other occupations and the general population (data demonstrate these rates for physicians [19], nurses [20], and veterinary medicine professionals [21]). Like the public's mounting interest in suicide prevention, the readiness within the house of medicine to address the drivers of suicide among health care professionals is also at a tipping point [22]. These twin topics - suicide prevention for patients and suicide prevention for health care providers - are indeed intertwined. Shedding stigma and caring for the mental health and suicide risk of health care providers can facilitate better care for the mental health and suicide risk of patients [23]. The American Medical Association, Accreditation Council for Graduate Medical Education, National Academy of Medicine, and nearly every other national health care association have made clinician wellbeing and suicide prevention a priority over the past 5 years $[24,25]$. Moreover, recently COVID-19's toll on the mental health and suicide risk of health care professionals has brought further urgency [26]. The death of Dr. Lorna Breen in April 2020 has been a lightning rod adding to these layers of readiness [27]. Never before has federal legislation been introduced that, if passed, would not only advocate for addressing clinicians' wellbeing and suicide but also provide appropriations to support and evaluate the outcomes of such efforts [28].

For human beings - including both patients and clinicianssuicide is a complex health outcome. Like any multi-dimensional health outcome, the best prevention strategies are based on evidence, multi-pronged, and sustained over time. In the June 2021 issue of Academic Psychiatry, two articles document the outcomes of programmatic suicide prevention efforts $[12,14]$. One of the articles [12] is an implementation of the American Foundation for Suicide Prevention's Interactive Screening Program [29], which affords faculty physicians and trainees the option to anonymously engage in a process of screening and personalized dialog that effectively moves high-risk individuals toward readiness to engage in treatment. Previous studies demonstrate that the vast majority of actively suicidal physicians and trainees are not in mental health treatment of any kind and have tremendous perceived and real barriers [30]. The second article [14] describes a peer-to-peer approach for medical students, which also has been utilized among high school and college students, because only half of suicidal youth disclose to anyone, and of those who disclose, two-thirds tell a peer [31].

\section{Clearing Up a Point About the Term Prevention in Suicide Prevention}

The phrase suicide prevention is used because science demonstrates that suicide is a generally preventable cause of death from a population health standpoint. In other words, investments at the national and local levels in policy, research, and programs can result in reductions in mortality, as has been demonstrated for other leading causes of death. Prevention is not the same as prediction, however, and conflating these terms often leads to incorrect 
assumptions, confusion, and guilt. While there is tremendous interest in the suicide research field looking into predicting near-term risk, only several years of scientific investigation have taken place, and the ability to predict suicidal behavior especially in the nearterm has not yet been achieved.

Thus, suicide as a "preventable" cause of death does not mean that every instance of suicide can be averted, nor that suicide is predictable, just as cardiologists and primary care providers do not take the meaning of prevention related to cardiac mortality to mean that they know who will die or when, nor to assume that no one with risk factors for heart disease who receives appropriate treatment and makes efforts with diet, exercise, and stress reduction will die of heart disease. From a public health standpoint, in the same way risk factors for cardiac mortality can be identified and addressed as an unequivocal way to reduce cardiac mortality risk, the same approach applies for reducing risk of suicide.

For the clinician whose patient dies by suicide and for family members whose loved one takes their life, the term prevention in suicide prevention does not mean they were at fault. Suicide is a complex, multi-dimensional health outcome in the same way that many complex health outcomes are. It is part of the normal grieving process and almost unavoidable during the aftermath of suicide to look back and hypothesize about potential moments of missed opportunity. Nevertheless, the need to do more to prevent suicide as a society and within health systems must not be confused with a narrative that places blame on any one moment, person, or risk factor. Suicide risk is too complex and multi-dimensional to be reduced to an overly simplistic cause-and-effect model.

Conversely, the lack of predictability does not mean that existing analytic models are not providing positive predictive values on par with acceptable actionable levels for other health issues. Several models for predicting suicide attempts have positive predictive values similar to or exceeding those of widely accepted risk tools for other health outcomes. For example, the Mental Health Research Network, comprising 13 large US health systems, is able to identify mental health outpatients with a $5 \%$ risk of suicidal behavior over the following 90 days [32]. For comparison, the US Preventive Services Task Force recommends statins for patients with predicted cardiac event exceeding $10 \%$ over 10 years [33] and recommends tamoxifen for women with a 3\% predicted breast cancer risk over 5 years [34]. Most clinicians work in settings where these suicide-related modeling tools are not yet in use, but these tools for the health system and electronic health records will likely be implemented at a wider scale in the near future.

\section{Postvention Is Prevention}

A truism in the suicide prevention field is that postvention is prevention. Its meaning is threefold. First, by facilitating healthy grieving for suicide loss survivors, the increased risk conferred by the experience of and exposure to suicide loss can be mitigated for suicide loss survivors [35]. Second, the potential for suicide contagion among the community following a suicide death can be mitigated by a coordinated response that communicates accurately and sensitively about the loss and provides maximal avenues for support, especially for the most vulnerable in the population. Third, suicide loss survivors have carried the torch in the movement of suicide prevention. Their passion to prevent other families from losing a loved one to suicide is a way for some to funnel grief into meaningful action and can provide a way to honor their loved one. Thankfully, as stigma has been diminishing, people with their own lived experience of a suicide attempt or a suicidal crisis have become a strong voice in the movement as well. (These categories of loss and lived experience are not mutually exclusive; many individuals may experience suicide loss as well as their own suicidal thoughts or behavior.) Therefore, the articles in this issue describing postvention efforts following patient suicide [15-18] are an important contribution for a comprehensive clinical suicide prevention effort in medical education. While the topic of postvention following a colleague or trainee's suicide is not specifically included in this issue, leaders are encouraged to utilize toolkits designed to provide guidance following a medical student, resident, or staff suicide [35].

\section{What Gives Me Hope?}

Science and education give me hope. Among the other top 10 leading causes of death, have any others previously been an orphan topic in medical education, only to evolve into a major focus for research and intervention later? In fact, there was a time when cancer was whispered in polite circles and no formal medical education was devoted to it [36]. Investments in science have been game changers for cancer, HIV/AIDS, heart disease, infectious disease, and other major public health threats, and the same will be true for mental health and suicide. The advances in the science of suicide risk and prevention, including the articles in this issue, give me hope. Academic medicine and psychiatry have a critical role to play in addressing suicide through educational curricula and programmatic efforts. Additionally, teachers lead by example first. By role modeling a no-stigma, my-own-mental-health-matters lifestyle, and by speaking out about making resilience a priority, health care leaders and educators send a powerful message [27]. Teaching suicide prevention science and skills, implementing curricula, and preventing suicide among health care workers during this period of elevated risk related to the pandemic can all contribute to the critical imperative of suicide prevention. 


\section{Declarations}

Disclosures The corresponding author is employed full-time by the organization (American Foundation for Suicide Prevention) that developed and licenses the Interactive Screening Program but does not receive monetary compensation related to the program or its implementation.

\section{References}

1. The Harris Poll. National survey shows majority of Americans would take action to prevent suicide. September 12, 2018. Available from: https://theharrispoll.com/national-survey-showsmajority-of-americans-would-take-action-to-prevent-suicide/. Accessed 11 March 2021.

2. Centers for Disease Control and Prevention. Mental health care: household pulse survey. March 24, 2021. Available from: https:// www.cdc.gov/nchs/covid19/pulse/mental-health-care.htm. Accessed 25 March 2021.

3. American Foundation for Suicide Prevention. Public policy priorities. 2021. Available from: https://afsp.org/public-policy-priorities. Accessed 11 March 2021.

4. Kochanek KD, Xu JQ, Arias E. Mortality in the United States, 2019. NCHS Data Brief, no 395. Hyattsville, MD: National Center for Health Statistics. 2020.

5. Hedegaard H, Curtin SC, Warner M. Increase in suicide mortality in the United States, 1999-2018. NCHS Data Brief, no 362. Hyattsville, MD: National Center for Health Statistics. 2020.

6. Wilcox HC, Kellam SG, Brown CH, Poduska JM, Ialongo NS, Wang W, et al. The impact of two universal randomized first- and second-grade classroom interventions on young adult suicide ideation and attempts. Drug Alcohol Depend. 2008;95(Suppl 1):S6073. https://doi.org/10.1016/j.drugalcdep.2008.01.005.

7. PR Newswire. The American Foundation for Suicide Prevention launches Phase II of Project 2025. Jan 15, 2019. Available from: https://www.prnewswire.com/news-releases/the-americanfoundation-for-suicide-prevention-launches-phase-ii-of-project2025-300778702.html. Accessed 16 Dec 2020.

8. Mann JJ, Michel CA, Auerbach RP. Improving suicide prevention through evidence-based strategies: a systematic review. Am J Psychiatry. 2021 Feb 18;appiajp202020060864. https://doi.org/10. 1176/appi.ajp.2020.20060864. Online ahead of print.

9. National Action Alliance for Suicide Prevention: Transforming Health Systems Initiative Work Group. Recommended standard care for people with suicide risk: making health care suicide safe. Washington, DC: Education Development Center, Inc.; 2018. Available from: https://theactionalliance.org/sites/default/files/ action_alliance_recommended_standard_care_final.pdf. Accessed 28 Dec 2020.

10. Chuop M, Michel Z, Shah R, Chen JI, Black W. From screening to interventions: teaching clinical suicide prevention skills to medical students. Acad Psychiatry. 2020 https://doi.org/10.1007/s40596020-01248-3. Online ahead of print.

11. Foster A, Alderman M, Safin D, Aponte X, McCoy K, Caughey M, et al. Teaching suicide risk assessment: spotlight on the therapeutic relationship. Acad Psychiatry. 2021 https://doi.org/10.1007/ s40596-021-01421-2. Online ahead of print.

12. Sciolla AF, Haskins J, Chang CH, Kirshnit C, Rea M, Uppington J, et al. The suicide prevention, depression awareness, and clinical engagement program for faculty and residents at the University of California, Davis Health. Acad Psychiatry. 2021. https://doi.org/10. 1007/s40596-021-01439-6 Online ahead of print.

13. Walukevich-Dienst K, Crapanzano KA, Zielinski MH, Vath RJ, Tucker RP. Integration of a suicide-specific treatment program within a psychiatric residency and large hospital system of care: a twelve-month journey. Acad Psychiatry. 2020. https://doi.org/10. 1007/s40596-020-01209-w Online ahead of print.

14. Eldridge A, Chen J, Furnari M, Makowichuk K, Black WE. Adopting a peer-to-peer approach to trainee suicide prevention. Acad Psychiatry. 2020 https://doi.org/10.1007/s40596-02001284-z. Online ahead of print.

15. Agrawal A, Gitlin M, Melancon SNT, Booth BI, Ghandhi J, DeBonis K. Responding to a tragedy: evaluation of a postvention protocol among adult psychiatry residents. Acad Psychiatry. 2021 https://doi.org/10.1007/s40596-021-01418-x. Online ahead of print.

16. Qayyum Z, AhnAllen CG, Van Schalkwyk GI, Luff D. "You really never forget it!" Psychiatry trainee supervision needs and supervisor experiences following the suicide of a patient. Acad Psychiatry. 2021 https://doi.org/10.1007/s40596-020-01394-8. Online ahead of print.

17. McCutcheon S, Hyman J. Increasing resident support following patient suicide: assessing resident perceptions of a longitudinal, multimodal patient suicide curriculum. Acad Psychiatry. 2021 https://doi.org/10.1007/s40596-021-01425-y. Online ahead of print.

18. Qayyum Z, Luff D, Van Schalkwyk GI, CG AA. Recommendations for effectively supporting psychiatry trainees following a patient suicide. Acad Psychiatry. 2021. https://doi. org/10.1007/s40596-020-01395-7 Online ahead of print.

19. Ye GY, Davidson JE, Kim K, Zisook S. Physician death by suicide in the United States: 2012-2016. J Psychiatr Res. 2021;134:158-65.

20. Davidson JE, Proudfoot J, Lee K, Terterian G, Zisook S. A longitudinal analysis of nurse suicide in the United States (2005-2016) with recommendations for action [Corrigendum: Worldviews Evid Based Nurs. 2020;17:180]. Worldviews Evid-Based Nurs. 2020;17(1):6-15.

21. Tomasi SE, Fechter-Leggett ED, Edwards NT, Reddish AD, Crosby AE, Nett RJ. Suicide among veterinarians in the United States from 1979 through 2015. J Am Vet Med Assoc. 2019;254: 104-12.

22. Feist JB, Feist JC, Cipriano P. Stigma compounds the consequences of clinician burnout during COVID-19: a call to action to break the culture of silence. NAM Perspectives. Commentary, National Academy of Medicine, Washington, DC. 2020. Available from: https://doi.org/10.31478/202008b. Accessed 7 January 2021

23. Moutier C. Physician mental health: an evidence-based approach to change. J Med Regul. 2018;104:7-13.

24. Smith TM. New resource advises how to prevent, respond to trainee suicide. American Medical Association. December 20, 2016. Available from: https:/www.ama-assn.org/residents-students/ resident-student-health/new-resource-advises-how-preventrespond-trainee-suicide. Accessed 3 January 2021.

25. National Academy of Medicine. Action collaborative on clinician well-being and resilience. 2021. Available from: https://nam.edu/ initiatives/clinician-resilience-and-well-being/. Accessed 14 January 2021.

26. Lai J, Ma S, Wang Y, Cai Z, Hu J, Wei N, et al. Factors associated with mental health outcomes among health care workers exposed to coronavirus disease 2019. JAMA Netw Open. 2020;3(3):e203976.

27. Moutier CY, Myers MF, Feist JB, Feist JC, Zisook S. Preventing clinician suicide: a call to action during the COVID-19 pandemic and beyond. Acad Med. 2021. https://doi.org/10.1097/ACM. 0000000000003972 Online ahead of print.

28. United States Congress. S.4349 - Dr. Lorna Breen Health Care Provider Protection Act. 116th Congress (2019-2020). Available from: https://www.congress.gov/bill/116th-congress/senate-bill/ 4349 ? $\mathrm{q}=\% 7 \mathrm{~B} \% 22 \mathrm{search} \% 22 \% 3 \mathrm{~A} \% 5 \mathrm{~B} \% 22 \mathrm{~d} \% 22 \% 5 \mathrm{D} \% 7 \mathrm{D} \& \mathrm{~s}=$ $1 \& \mathrm{r}=3$. Accessed 11 March 2021.

29. American Foundation for Suicide Prevention. ISP for medical schools, hospitals and health systems . 2021. Available from: 
https://afsp.org/isp-for-medical-schools-hospitals-and-healthsystems. Accessed 11 March 2021.

30. Mortali M, Moutier C. Facilitating help-seeking behavior among medical trainees and physicians using the interactive screening program. J Med Regul. 2018;104:27-36.

31. Drum DJ, Brownson C, Burton Denmark A, Smith SE. New data on the nature of suicidal crises in college students: shifting the paradigm. Prof Psychol Res Pract. 2009;40:213-22.

32. Simon GE, Shortreed SM, Coley RY. Positive predictive values and potential success of suicide prediction models. JAMA Psychiatry. 2019;76(8):868-9.

33. Preventive Services Task Force US, Bibbins-Domingo K, Grossman DC, Curry SJ, Davidson KW, Epling JW Jr, et al. Statin use for the primary prevention of cardiovascular disease in adults: US Preventive Services Task Force Recommendation statement. JAMA. 2016;316(19):1997-2007.
34. Preventive Services Task Force US, Owens DK, Davidson KW, Krist AH, Barry MJ, Cabana M, et al. Medication use to reduce risk of breast cancer: US Preventive Services Task Force Recommendation Statement. JAMA. 2019;322(9):857-67.

35. Erlangsen A, Pitman A. Effects of suicide bereavement on mental and physical health. In K. Andriessen, K. Krysinska, \& O. T. Grad (Eds.), Postvention in action: the international handbook of suicide bereavement support (pp. 17-26). Boston, MA: Hogrefe Publishing. 2017.

36. Payne S, Burke D, Mansi J, Jones A, Norton A, Joffe J, et al. Discordance between cancer prevalence and training: a need for an increase in oncology education. Clin Med. 2013;13:50-6.

Publisher's Note Springer Nature remains neutral with regard to jurisdictional claims in published maps and institutional affiliations. 